\title{
Characteristics of the Vertically Heterogeneous and Anisotropic Plate Structure in the Pacific: Implication of the Plate Thickness
}

\author{
Mitsuru Yoshida \\ Earthquake Research Institute, The University of Tokyo, Tokyo 113, Japan
}

\begin{abstract}
A detailed shear wave velocity structure in the Pacific lithosphere for an ocean-floor age greater than $100 \mathrm{~m}$.y. has been obtained in high resolution by means of several isotropic and anisotropic surface wave inversions. The vertically heterogeneous velocity structure of the vertically polarized shear wave has the following 3 characteristics: (1) has a small-scale low velocity zone near a depth of $30 \mathrm{~km}$; (2) reaches a maximum value at a depth of $70 \mathrm{~km}$; (3) obtains a minimum value at a depth of $130 \mathrm{~km}$ in the large-scale low velocity zone. The velocity increases with depth between $130-220 \mathrm{~km}$. The velocity structure of the horizontally polarized shear wave does not possess Characteristics 1 and 2. Characteristics 2 and 3 give strong constraints on the rigorous estimation of the plate thickness. If the bottom of the heterogeneous lithosphere is tentatively defined as a mid-point between the depths indicating Characteristics 2 and 3, the plate thickness is estimated to be $100 \mathrm{~km}$ for the region where the age is greater than 100 m.y. A relationship between Characteristic 1 and the double-planed seismic zone is also discussed.
\end{abstract}

\section{Introduction}

After applying an anelasticity correction to surface wave data, the regional structure of the Pacific lithosphere and asthenosphere has been constructed through the use of several modern inversion techniques. These techniques have been used by a number of investigators (Mitchell and Yu, 1980; Regan and Anderson, 1984; Kawasaki, 1986; Yoshida, 1986; Nishimura and Forsyth, 1989). With respect to the plate thickness, a number of the lithospheric structures are classified according to either a thick model or a thin model. The plate thickness is compared with the elastic thickness inferred from studies of the lithospheric flexure (Watts et al., 1980; Ida, 1984). The elastic thickness is estimated to be between $30-60 \mathrm{~km}$ (Watts et al., 1980) for the ocean floor with an age of $150 \mathrm{~m} . \mathrm{y}$.

If the thickness estimated by surface waves is designated as the seismic thickness, it is found that the thickness is $2-3$ times greater than the elastic thickness. It should be noted that the regional models developed by various authors using surface waves for the same region show distinct differences in the structure of the lithosphere, the

Received January 25, 1990; Accepted May 11, 1990 
asthenosphere, or both. This is due to the fact that both the thick and thin models involve some errors and uncertainties associated with uniqueness and/or resolution. Therefore, the background for the solutions should be critically studied in order to correctly interpret the solutions.

The present paper attempts to obtain a vertically heterogeneous shear wave velocity structure in the Pacific lithosphere and asthenosphere for a region where the age is greater than 100 m.y. The generalized inverse method (Wiggins, 1972; Jackson, 1972) is applied to several anisotropic and isotropic inversions. Trade-off curves are calculated in order to select the optimized solution defined in terms of the generalized eigenvectors. The heterogeneous lithospheric structure will create a new image of the plate model that will then afford extensive views of seismic thickness and the double-planed seismic zone.

\section{Thick and Thin Models}

The plate model developed by Mitchell and Yu (1980) having a thickness of $100 \mathrm{~km}$ for the region where the age is $>100 \mathrm{~m}$.y. is selected as the thick model. This model is modified by assuming a fixed value of $4.20 \mathrm{~km} / \mathrm{s}$ in the low velocity zone (LVZ) and a fixed depth of $180 \mathrm{~km}$ for the bottom of the LVZ. The starting model used by Mitchell and $\mathrm{Yu}$ had a plate thickness of $110 \mathrm{~km}$ corresponding to the region having an age of $120 \mathrm{~m} . \mathrm{y}$. in the very thick model obtained by Leeds et al. (1974). The very thick model has a plate thickness of $150 \mathrm{~km}$ in the region where the age is $150 \mathrm{~m} . \mathrm{y}$.

Thin models with a thickness of $60 \mathrm{~km}$ for the region where the age is $>100 \mathrm{~m}$.y. have been developed by Anderson and Regan (1983), Regan and Anderson (1984), and Kawasaki (1986), making use of anisotropic inversions of the data sets of Mitchell and $\mathrm{Yu}$ (1980). A distinct feature of the three thin models is that the shear wave velocity structure in the LVZ exhibits a drastic change between young and old regions of the Pacific. It should also be noted that prominent differences exist between the models of Regan and Anderson (1984) and those of Kawasaki (1986), particularly in the structure of the LVZ in the region where the age is $>100$ m.y., and for other regions, as will be discussed below.

\section{Calculation of the Detailed Structure of the Lithosphere and Asthenosphere}

\subsection{Data}

The data sets consisting of the group and phase velocities of Rayleigh and Love waves in the regions where the age is $>1.00 \mathrm{~m}$.y. and between $50-100 \mathrm{~m} . \mathrm{y}$., as reported by Mitchell and Yu (1980), are used in the present anisotropic and isotropic inversions. In order to check the nonuniqueness of the resulting structures, data sets consisting of group velocities of Rayleigh waves in the adjacent areas, i.e., >150 m.y. (Yoshida, 1978), >90 m.y. and between 50-90m.y. (Yoshii, 197.5), are used in isotropic inversions. The data sets of group velocities in the young region of 20-50 m.y. (Yoshii, 1975; Mitchell and Yu, 1980) are also used in isotropic inversions for comparison purposes. The anelasticity correction (Liu et al., 1976; Kanamori and Anderson, 1977) is performed on the above data sets at a reference period of $1 \mathrm{~s}$, using the $\mathrm{Q}$ data of 
Mitchell (1976). The period of waves in the data sets ranges from 30 to $110 \mathrm{~s}$.

The oceanic model 8099 presented by Dorman et al. (1960) and the global model PREM (Dziewonski and Anderson, 1981), which greatly differs from the 8099 model at depths shallower than $220 \mathrm{~km}$, are employed as starting models. The 8099 model is used without the addition of any modifications. The PREM model is slightly modified for layer parameters at depths shallower than $220 \mathrm{~km}$. These modifications consist of constant densities of 3.3791 and $3.3682 \mathrm{~g} / \mathrm{cm}^{3}$ at depths between $11.4-60$ and $60-220 \mathrm{~km}$, respectively, while compressional wave velocities of 8.1012 and $8.0399 \mathrm{~km} / \mathrm{s}$ are assumed for depths between $11.4-60$ and $60-220 \mathrm{~km}$, respectively. For the structure of shear wave velocity, the values of the 8099 model are used while the crustal structure employs the 3 layer parameters of Cara (1979). A water depth of $5 \mathrm{~km}$ is assumed in both starting models. The two models are used to check the nonuniqueness of the resulting structures due to starting-model dependence.

\subsection{Method}

The partial derivatives of the group and phase velocities are employed in the present linearized inversion. The theoretical relationship between these derivatives, as shown by Rodi et al. (1975), is given by

$$
\left.\frac{\partial U}{\partial m}\right|_{\omega}=\left.\frac{U_{v}}{C}\left(2-\frac{U}{C}\right) \frac{\partial C}{\partial m}\right|_{\omega}+\left.\omega \frac{U^{2}}{C^{2}} \frac{\partial}{\partial \omega}\left(\left.\frac{\partial C}{\partial m}\right|_{\omega}\right)\right|_{m}
$$

and

$$
\left.\frac{m}{U} \frac{\partial U}{\partial m}\right|_{\omega}=\frac{m}{C} \frac{\partial C}{\partial m}+\left.\frac{U}{C} \omega \frac{\partial}{\partial \omega}\left(\left.\frac{\omega}{C} \frac{\partial C}{\partial m}\right|_{\omega}\right)\right|_{m} .
$$

Here, $U$ and $C$ are the group and phase velocities at a given frequency $\omega ; \partial U /\left.\partial m\right|_{\omega}$ and $\partial C /\left.\partial m\right|_{\omega}$ are the partial derivatives of the group and phase velocities with respect to a layer parameter $m$, taken while holding $\omega$ fixed.

The partial derivatives of the phase velocity can be obtained through use of the energy integrals given in Takeuchi and Saito (1972) as follows:

$$
\frac{\beta_{\mathrm{H}}}{C}\left[\frac{\partial C}{\partial \beta_{\mathrm{H}}}\right]_{\omega, \rho, \beta \vee}=\frac{1}{\omega^{2} I_{1}^{\mathrm{L}}}\left(\frac{U}{C}\right) k^{2} N\left(y_{1}^{\mathrm{L}}\right)^{2}
$$

and

$$
\frac{\beta_{\mathrm{V}}}{C}\left[\frac{\partial C}{\partial \beta_{\mathrm{V}}}\right]_{\omega, \rho, \beta_{\mathrm{H}}}=\frac{1}{\omega^{2} I_{1}^{\mathrm{R}}}\left(\frac{C}{U}\right)\left(\frac{1}{L}\left(y_{4}^{\mathrm{R}}\right)^{2}+\frac{4 k F L}{A-2 L} \dot{y}_{1}^{\mathrm{R}} y_{3}^{\mathrm{R}}\right),
$$

where the superscripts $\mathrm{L}$ and $\mathrm{R}$ denote Love and Rayleigh waves, respectively. $I_{1}^{\mathrm{L}}$ and $I_{1}^{\mathrm{R}}$ represent the energy integrals. $\beta_{\mathrm{H}}$ and $\beta_{\mathrm{V}}$ are the horizontally and vertically polarized shear wave velocities, respectively, while $\rho$ is the density and $k$ is the wave number. $N$, $L, F$, and $A$ are elastic constants, with $y_{1}^{\mathbf{R}}, y_{2}^{\mathbf{R}}, y_{3}^{\mathbf{R}}$, and $y_{4}^{\mathbf{R}}$ representing the eigenfunctions; $\dot{y}_{1}^{\mathrm{R}}$ stands for the derivative of $y_{1}^{\mathrm{R}}$ with respect to depth.

The generalized inverse method (Wiggins, 1972; Jackson, 1972), weighted by the

Vol. 38, No. 1, 1990 
layer thickness, is employed in order to determine the model parameters. The optimized solution $\hat{\boldsymbol{x}}$ is obtained by

$$
\hat{\boldsymbol{x}}=A_{q}^{\dagger} \boldsymbol{y} .
$$

Here, $y$ is the data vector, and the generalized inverse of the coefficient matrix

$$
A_{q}^{\dagger}=V_{q} \Lambda_{q}^{-1} U_{q}^{T}
$$

is an arbitrary $m \times n$ matrix of rank $q(<n, m) . \boldsymbol{A}_{q}^{-1}$ is a semidiagonal $q \times q$ matrix of singular values. $V_{q}$ and $U_{q}^{T}$, formed from the eigenvectors $\boldsymbol{v}_{i}$ and $\boldsymbol{u}_{i}(i=1, \cdots, q)$, are an orthonormal $m \times q$ matrix and a transposed orthonormal $q \times n$ matrix, respectively. Following Yoshida (1986), the number of the largest singular values (lsv), degree of freedom in the data, is determined in such a way as to optimize the combination of the criteria given below (e.g., see Jackson, 1972; Saito, 1983).

$$
\begin{aligned}
& R_{q}=A_{q}^{\dagger} A_{q}=V_{q} V_{q}^{T}=r_{i j} \sim I_{m}, \\
& S_{q}=A_{q} A_{q}^{\dagger}=U_{q} U_{q}^{T}=s_{i j} \sim I_{n}
\end{aligned}
$$

and

$$
\sigma_{k}^{2}=\operatorname{var}\left(\hat{x}_{k}\right)=\sum_{j=1}^{q}\left(v_{k, j} / \lambda_{j}\right)^{2} \sim \text { small }(<0.04 \mathrm{~km} / \mathrm{s}),
$$

where $\lambda_{j}$ is the singular value. $\boldsymbol{R}$ and $S$ are the resolution matrix and the information matrix, respectively, while $\sigma_{k}^{2}$ is the variance of $\hat{x}_{k}$.

In order to determine the proper choice of the optimized solution, the following five quantities are calculated, which include the criteria mentioned above.

(i) The average perturbations of the unknown parameters in percent,

$$
\overline{X P}=\sum_{i=1}^{m}\left|\Delta x_{i} / x_{i}\right| \times 100 / m,
$$

where $x_{i}$ is the initial value and $\Delta x_{i}$ the perturbation.

(ii) The root mean square of the group (or phase) velocity residuals, given by

$$
\overline{O C}=\sqrt{\sum_{i=1}^{n}\left|O_{i}-C_{i}\right|^{2} / n},
$$

where $O_{i}$ and $C_{i}$ are the observed and calculated group (or phase) velocities for the $i$ th component, respectively.

(iii) The average of the standard deviations

$$
\overline{S D}=\sum_{i=1}^{m}\left|\sigma_{i}\right| / m,
$$

where $\sigma_{i}$ is the standard deviation of the $i$ th component. For the following two quantities, the resolution matrix Eq. (7) and information matrix Eq. (8) are normalized by the maximum value in each row.

(iv) The measure of the resolution matrix. 


$$
\bar{R}=\sum_{i=1}^{m} \sum_{j=1}^{m}\left|r_{i j}-\delta_{i j}\right| / m^{2}
$$

where $\delta_{i j}$ is the Kronecker's delta. If $\bar{R}=0$, then the solution is uniquely determined.

(v) The measure of the information matrix, given by

$$
\bar{I}=\sum_{i=1}^{n} \sum_{j=1}^{n}\left|s_{i j}-\delta_{i j}\right| / n^{2} \text {. }
$$

If $\bar{I}=0$, then the data is highly independent.

The partial derivatives of surface wave velocities with respect to physical parameter changes (Yoshida, 1986) show that the shear wave velocity influences most strongly the dispersion characteristics of suface waves. Therefore, in the present work, the measure $\bar{R}$ for only the $\beta_{\mathrm{V}}-\beta_{\mathrm{V}}$ or the $\beta_{\mathrm{H}^{-}} \beta_{\mathrm{H}}$ is shown in the trade-off curves.

\section{Results and Interpretation}

4.1 The $\beta_{\mathrm{V}}$ and $\beta_{\mathrm{H}}$ structures in the region $>100$ m.y.

First, two types of isotropic inversions were carried out using the Rayleigh wave

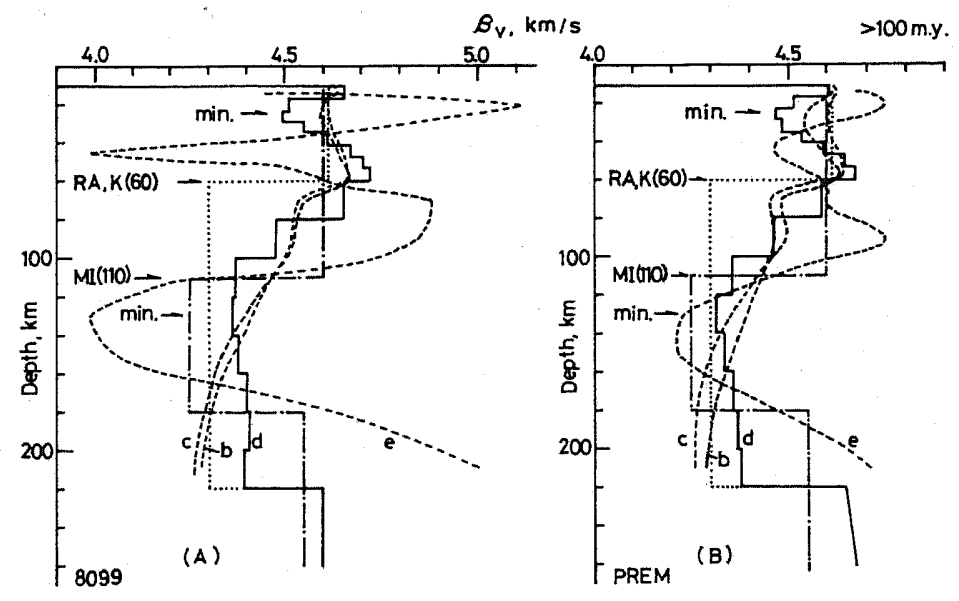

Fig. 1. Shear wave velocity structures (A) and (B) in the region $>100 \mathrm{~m} . \mathrm{y}$. obtained from Rayleigh wave group velocities, using the 8099- and PREM-type starting models, respectively. Structures of the starting models are denoted by dotted lines and the one used by Mitchell and Yu (1980) by dash-dot lines. The structures b, c, d, and e correspond to the solutions for $q=2,3,4$, and 5 , respectively, ( $q$ : the number of the largest singular values). The arrows with the abbreviation min. indicate depth taking a minimum velocity. The plate thicknesses, denoted by numerals in parentheses, are estimated from the models RA (Regan and Anderson, 1984), K (Kawasaki, 1986), and MI (Mitchell and $\mathrm{Yu}, 1980)$. They are marked by arrows with their model abbreviations. Note that the number and kind of parameters solved are different for structures (A) and (B) (See the text for details).

Vol. 38, No. 1, 1990 

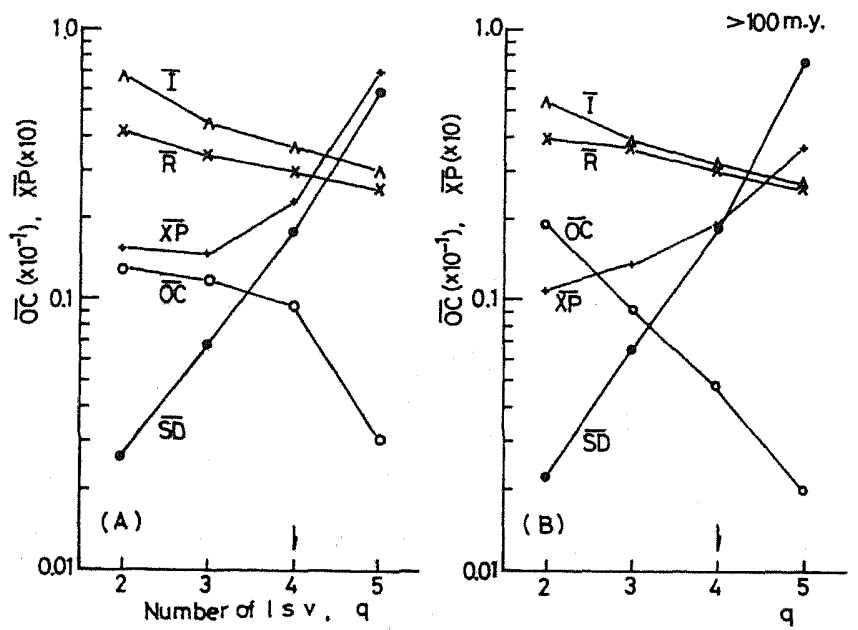

Fig. 2. Trade-off curves between the perturbations of parameters $(\overline{X P})$, group velocity residuals $(\overline{O C})$, standard deviations $(\overline{S D})$, resolutions $(\bar{R})$, and information densities $(\bar{I})$ for the structures $(\mathrm{A})$ and $(\mathrm{B})$ given in Fig. 1. The vertical arrow on the abscissa indicates the number $(q)$ of the largest singular values (lsv) that yields the optimized solution.

group velocities of Mitchell and Yu (1980) for the region where the age is $>100 \mathrm{~m} . \mathrm{y}$. The structures (A) and (B), given in Fig. 1, were obtained by using the 8099- and PREM-type starting models, respectively. For the former case, only the $\beta_{\mathrm{V}}$ was determined, while in the latter the density $\rho, \beta_{\mathrm{V}}$, vertically $\left(\alpha_{\mathrm{V}}\right)$ and horizontally $\left(\alpha_{\mathrm{H}}\right)$ polarized compressional wave velocities along with the anisotropic factor $\eta$ were simultaneously determined.

The trade-off curves, calculated on the basis of the expressions given by Eqs. (10)-(14), are shown in Fig. 2. The values of $\overline{X P}, \overline{O C}, \overline{S D}, \bar{R}$, and $\bar{I}$ at $q=4$ for Case A were determined as approximately $2.2 \%, 0.01 \mathrm{~km} / \mathrm{s}, 0.18 \mathrm{~km} / \mathrm{s}, 0.3$, and 0.4 , respectively. The values of $\overline{S D}$ at $q=5$, for Cases $\mathrm{A}(=0.6)$ and $\mathrm{B}(=0.7)$, are too large, while those of $\bar{R}, \bar{I}$, and $\overline{O C}$ become small. It should be noted that the $\overline{X P}$ curve is increasing more rapidly with the increase in $q$ for Case A than for Case B. Taking the trade-off curves into consideration, two structures, determined by the d models $(q=4)$ for Cases $A$ and $B$, are candidates for the region $>100 \mathrm{~m} . \mathrm{y}$. The $\mathrm{d}$ models possess three characteristics: 1) a small-scale LVZ at a depth of $30 \mathrm{~km}$ in the lithosphere; 2) a maximum velocity at a depth of $60 \mathrm{~km} ; 3)$ a minimum velocity at a depth of $130 \mathrm{~km}$. The resolutions (Fig. 3(a)) at depths of $30,70,130$, and $170 \mathrm{~km}$ are very high for Cases A and B. The characteristics of the information densities (Fig. 3(b)) for Case A are very similar to those for Case B. The group velocity residuals, shown in Fig. 3(c), are somewhat larger for Case A than for Case B.

Second, in order to check the validity of the d models shown in Fig. 1, the structures for neighboring areas (Fig. 4) were determined using the Rayleigh wave group velocities of Yoshii (1975) and Yoshida (1978) for the regions $>90$ m.y. and >150m.y., 


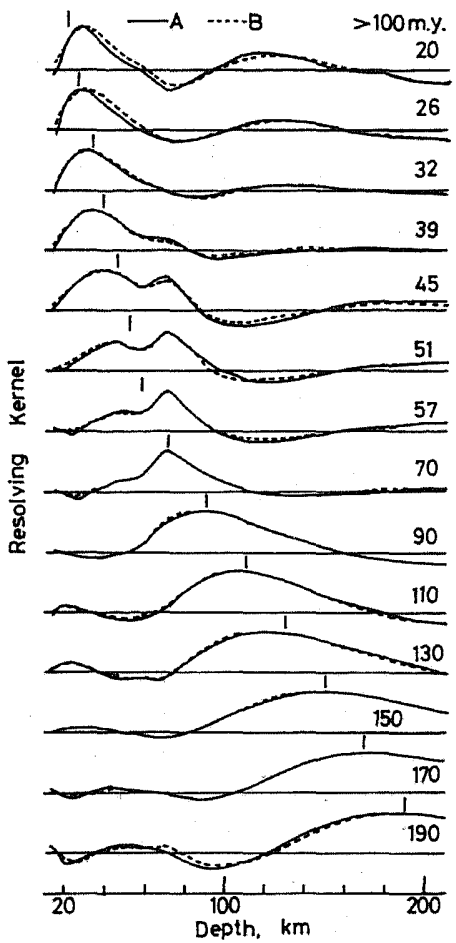

(a)

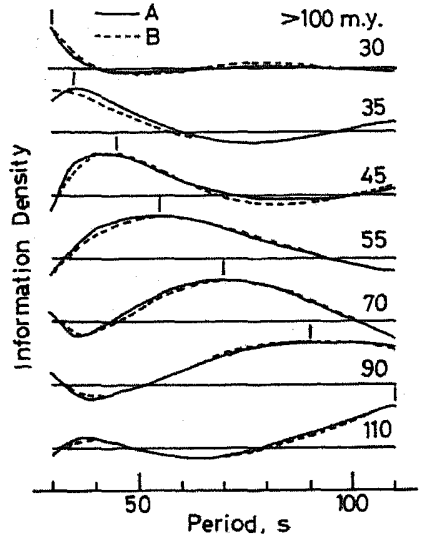

(b)

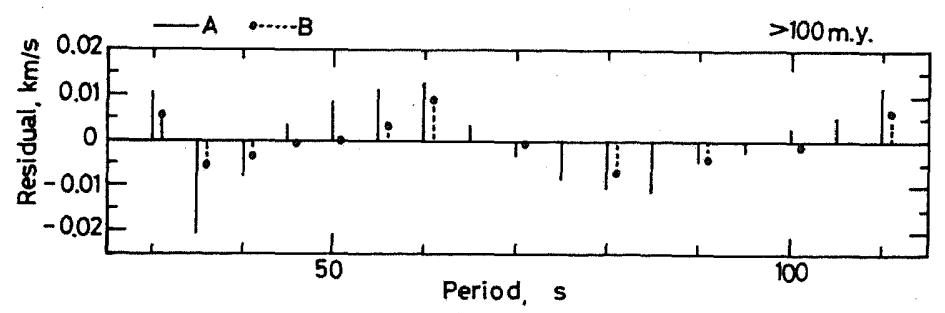

(c)

Fig. 3. Resolving kernels for the shear wave velocities (a), information densities (b), and group velocity residuals (c) calculated for the d models $(q=4)$ of structures (A) (solid lines) and (B) (dotted lines) given in Fig. 1.

respectively. The 8099-type starting model was used for the inversion. The trade-off curves (Fig. 5) show that the variations of $\overline{X P}, \overline{O C}$, and $\overline{S D}$ curves versus $q$ are not as great when compared with those in Fig. 2. The values of $\overline{S D}$ at $q=5$ are within $0.2 \mathrm{~km} / \mathrm{s}$ for the two regions. The resolutions, information densities, and group velocity residuals (Fig. 6) for the $\mathrm{d}(q=4)$ and e $(q=5)$ models show that three measures for the latter model are superior to the former. The $\beta_{\mathrm{v}}$ structures obtained (the e models in Fig. 4) are essentially similar to the $d$ models for the region $>100$ m.y. in Fig. 1.

Third, anisotropic inversions were carried out using both group and phase velocity data of Rayleigh and Love waves of Mitchell and $\mathrm{Yu}(1980)$ in the regions $>100 \mathrm{~m}$.y.

Vol. 38, No. 1, 1990 


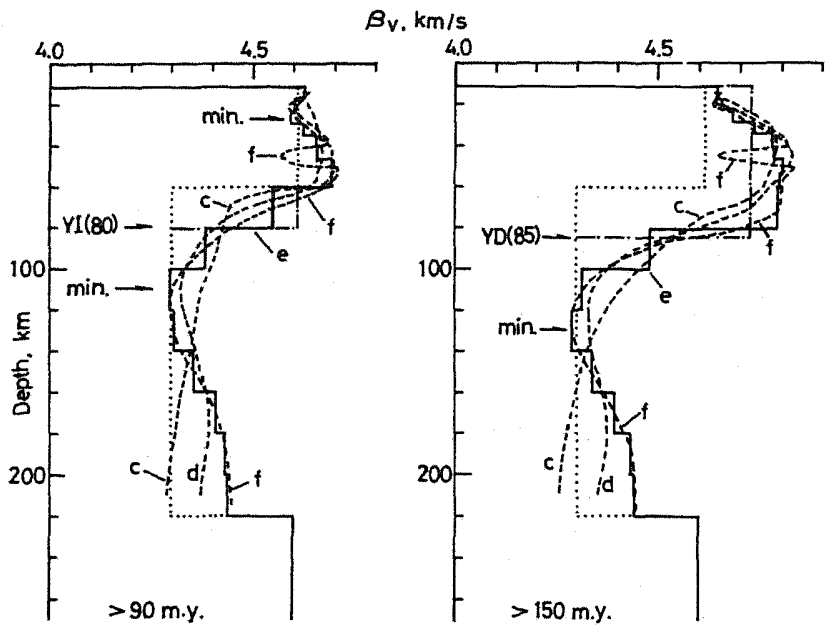

Fig. 4. Shear wave velocity structures in the regions $>90$ m.y. and $>150$ m.y. obtained from Rayleigh wave group velocities, using the 8099-type starting model (dotted lines). The structures $\mathrm{c}, \mathrm{d}, \mathrm{e}$, and $\mathrm{f}$ correspond to the solutions for $q=3,4,5$, and 6 , respectively. The plate thicknesses denoted by numerals in parentheses, estimated by YI (Yoshii, 1975) and YD (Yoshida, 1978), are marked by arrows with their model abbreviations.

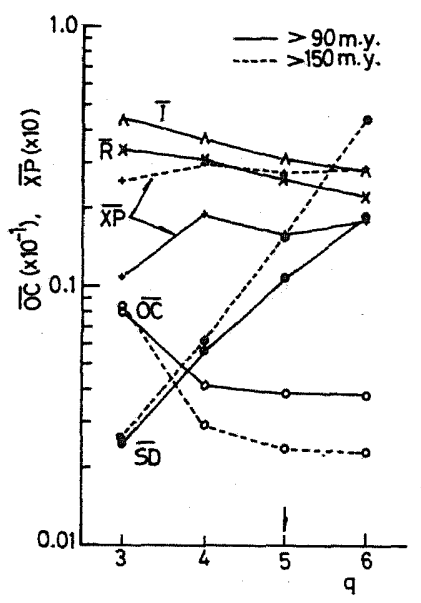

Fig. 5. Trade-off curves for the structures in the regions $>90 \mathrm{~m}$.y. (solid lines) and $>150$ m.y. (dotted lines) given in Fig. 4.

and between 50-100 m.y. It was assumed that the lithosphere and asthenosphere are transversely isotropic with a vertical symmetric axis. The PREM-type model was used as the starting model, in which the $\beta_{\mathrm{H}}$ and $\beta_{\mathrm{V}}$, as well as the $\alpha_{\mathrm{H}}$ and $\alpha_{\mathrm{V}}$, are assumed to be equal. Even though the six parameters $\left(\beta_{\mathrm{v}}, \beta_{\mathrm{H}}, \alpha_{\mathrm{V}}, \alpha_{\mathrm{H}}, \rho\right.$, and $\left.\eta\right)$ were simultaneously 

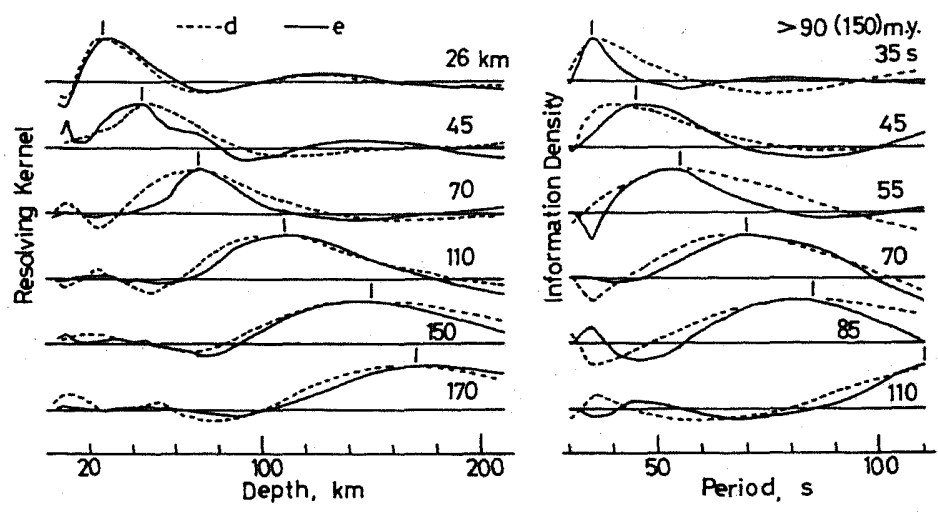

(a)

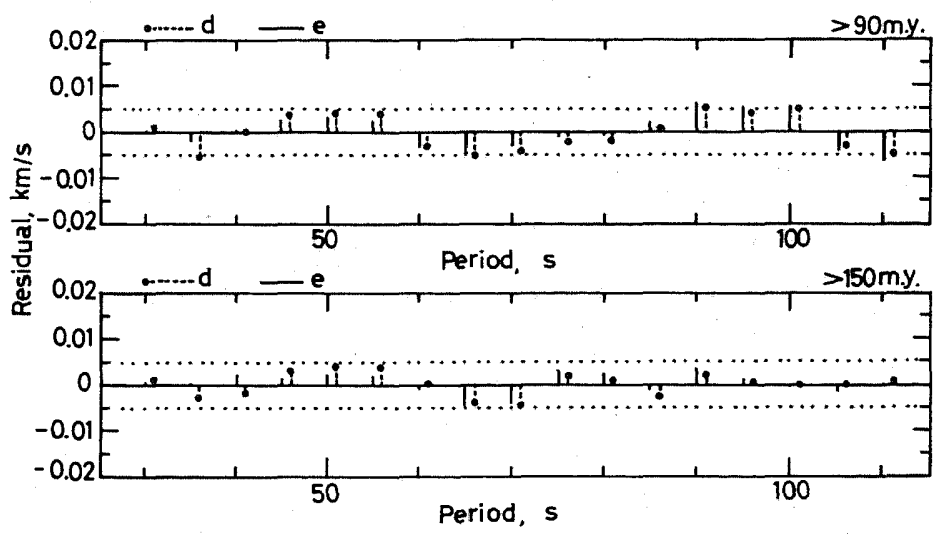

(b)

Fig. 6. Resolving kernels and information densities (a) and group velocity residuals (b) for the d(dotted lines) and e (solid lines) models given in Fig. 4.

determined in these inversions, the structures of only the $\beta_{\mathbf{V}}$ and $\beta_{\mathbf{H}}$ are shown in Fig. 7(a), since the focus here was concentrated on the shear wave velocity. The trade-off curves (Fig. 7(b)) show that the values of $\overline{S D}$ of the $\beta_{\mathrm{H}}$ are always smaller than those of the $\beta_{\mathrm{V}}$, while the values of $\bar{R}$ are reversed at $q \geq 3$.

The $\beta_{\mathrm{V}}$ structure for the region $>100 \mathrm{~m}$.y., obtained at $q=4$ from the group velocities, is very similar to the d models $(q=4)$ in Fig. 1 . It possesses the three characteristics previously described but Characteristic 2 should be modified in that the structure has a maximum velocity at a depth of $70 \mathrm{~km}$. It should be noted that the $\beta_{\mathrm{v}}$ increases with depth, being larger in the structure shown in Fig. 7(a) than in the $d$ models in Fig. 1 at depths between $130-220 \mathrm{~km}$. In the $\beta_{\mathrm{V}}$ structures, the locations that indicate the three characteristics derived from phase velocities occur at somewhat deeper depths than those derived from the group velocities.

The characteristic of the $\beta_{\mathrm{v}}$ structure for the region 50 to $100 \mathrm{~m} . \mathrm{y}$., derived from

Vol. 38 , No. 1,1990 

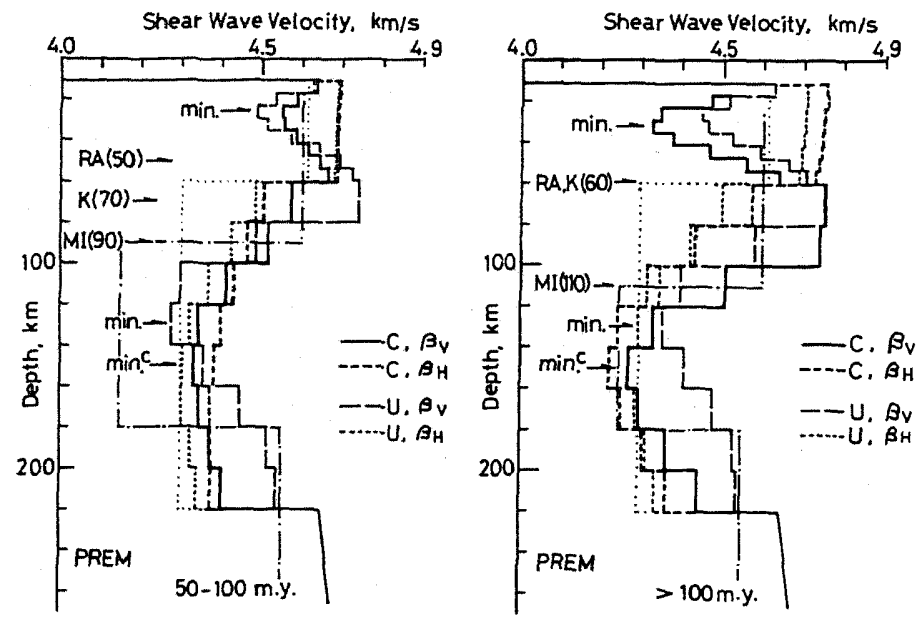

(a)
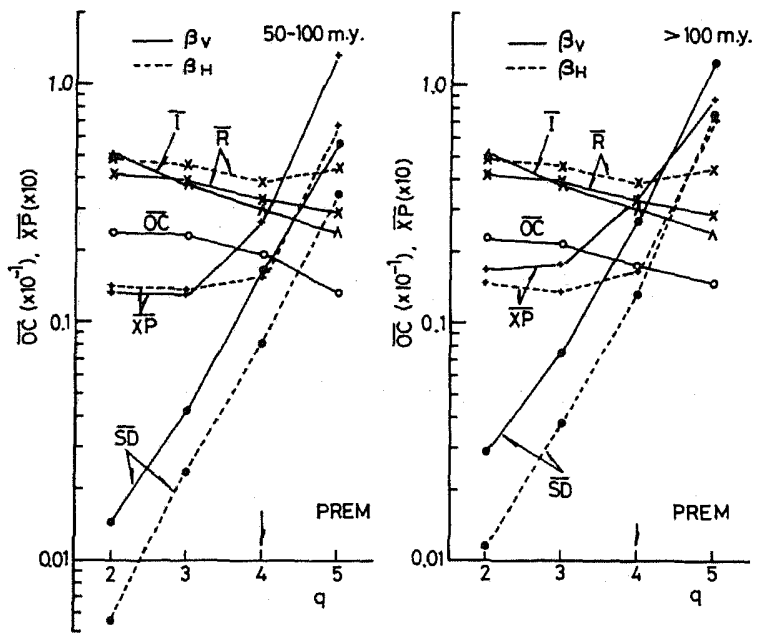

(b)

Fig. 7. (a) The $\beta_{\mathrm{V}}$ and $\beta_{\mathrm{H}}$ structures in the regions 50 to $100 \mathrm{~m} . \mathrm{y}$. and $>100 \mathrm{~m}$.y., obtained from anisotropic inversions, using the PREM-type starting model (dotted lines). The structure of the starting model used by Mitchell and $\mathrm{Yu}$ (1980) is indicated by chain lines (MI). The structures obtained from phase and group velocities are denoted by $C$ and $U$, respectively: The former is indicated by thick solid $\left(\beta_{\mathrm{V}}\right)$ and dotted $\left(\beta_{\mathrm{H}}\right)$ lines; the latter by thin broken $\left(\beta_{\mathrm{V}}\right)$ and dotted $\left(\beta_{\mathrm{H}}\right)$ lines. The arrows with the abbreviation min. ${ }^{\mathrm{c}}$ denote the depths for a minimum velocity in the large-scale LVZ of the $\beta_{\mathrm{V}}$ structures inferred from phase velocities. (b) Trade-off curves for the $\beta_{\mathrm{V}}$ and $\beta_{\mathrm{H}}$ structures in the regions 50 to 100 m.y. and $>100$ m.y. obtained from group velocities of Rayleigh and Love waves. The measures for the $\beta_{\mathrm{V}}$ and $\beta_{\mathrm{H}}$ structures are denoted by solid and dotted lines, respectively. For an explanation of the vertical arrows on the abscissa, see the caption in Fig. 2. 
group velocities, is close to that for the region $>100$ m.y. (Fig. 7(a)). This agreement holds except that the minimum velocity at a depth of $130 \mathrm{~km}$ in the large-scale LVZ for the former is lower by about $0.1 \mathrm{~km} / \mathrm{s}$ than for the latter. One of the most important characteristics of the $\beta_{\mathrm{H}}$ structures derived from the phase and group velocities, both for the regions $50-100 \mathrm{~m} . \mathrm{y}$. and $>100 \mathrm{~m} . \mathrm{y}$., is the absence of the small-scale LVZ at a depth of $30 \mathrm{~km}$. Accordingly, the large polarization anisotropy $\left(\beta_{\mathrm{H}}>\beta_{\mathrm{V}}\right)$ is clearly found in the region near that depth. It is also noted that the $\beta_{\mathrm{H}}$ is significantly higher in the lithosphere than in the entire asthenosphere.

\subsection{The vertically heterogeneous $\beta_{\mathrm{V}}$ structure and several plate models with uniform thickness}

For the $\beta_{\mathrm{v}}$ profiles in the region $>100 \mathrm{~m}$.y. (Figs. 1 and 7), the plate thicknesses estimated by several investigators have been indicated by arrows along with the model abbreviation: RA (Regan and Anderson, 1984), K (Kawasaki, 1986), and MI (Mitchell and $\mathrm{Yu}, 1980$ ). The vertically heterogenous $\beta_{\mathrm{v}}$ structure obtained in the present work reaches, as previously described, a maximum value at a depth of $70 \mathrm{~km}$ (Characteristic 2) and a minimum value at a depth of $130 \mathrm{~km}$ (Characteristic 3). These two characteristics give objective constraints on the determination of plate thickness.

Three $\beta_{\mathbf{V}}$ structures, representing the model obtained in the present study, along with the thin and thick models, are schematically illustrated in Fig. 8. Here, the lithosphere of the first model is subdivided into layers I, II, III, and IV. The subdivided layers appear to give useful indicators for comparison among the three plate models. The bottom of the thin plate model, denoted by RA and $\mathrm{K}$ for the region $>100 \mathrm{~m}$.y., is located at the boundary between layers II and III. This boundary, however, corresponds to the upper parts of the highest $\beta_{\mathrm{V}}$, so the plate thickness of $60 \mathrm{~km}$ estimated by the thin model seems to be underestimated.

The plate bottom of the thick model, denoted by MI for the region $>100 \mathrm{~m} . \mathrm{y}$., is located in the lower portion of layer IV, rather close to $130 \mathrm{~km}$, where the velocity reaches a minimum. However, a plate thickness of $110 \mathrm{~km}$ estimated by the thick model cannot be determined uniquely, since layer IV is transitional with the velocity decreasing downward.

When dealing with a vertically heterogeneous plate structure, a proper definition of the plate bottom is needed to define the plate thickness. If the plate is simply defined as the location that is indicated by Characteristic 2 or 3 , the thickness is estimated to be 70 or $130 \mathrm{~km}$. If the plate bottom is tentatively defined as the mid-point between the depths indicating these characteristics, the thickness can be estimated as $100 \mathrm{~km}$.

It should be noted that the differences in the $\beta_{\mathrm{V}}$ structures between the two thin models, denoted by RA and $\mathrm{K}$, are remarkable for both the regions $>100 \mathrm{~m}$.y. and 50 to 100 m.y. The RA model is relatively close to the $\mathrm{b}(q=2)$ or $\mathrm{c}(q=3)$ models shown in Figs. 1 and 4. This implies that the RA model is small in $\overline{S D}$ but has large $\bar{R}$ compared with the heterogeneous $\mathrm{d}(q=4)$ and e $(q=5)$ models.

The elastic thickness $\left(T_{\mathrm{e}}\right)$, schematically indicated in Fig. 8, is too thin when compared with the seismic thickness $\left(T_{\mathrm{s}}\right)$. However, as previously revealed in Fig. 7(a), the $\beta_{\mathrm{H}}$ structures in the region $>100 \mathrm{~m}$.y. are significantly different from the $\beta_{\mathrm{V}}$ structures. The $\beta_{\mathrm{H}}$ reaches its maximum in the uppermost parts of the lithosphere, decreasing

Vol. 38 , No. 1,1990 


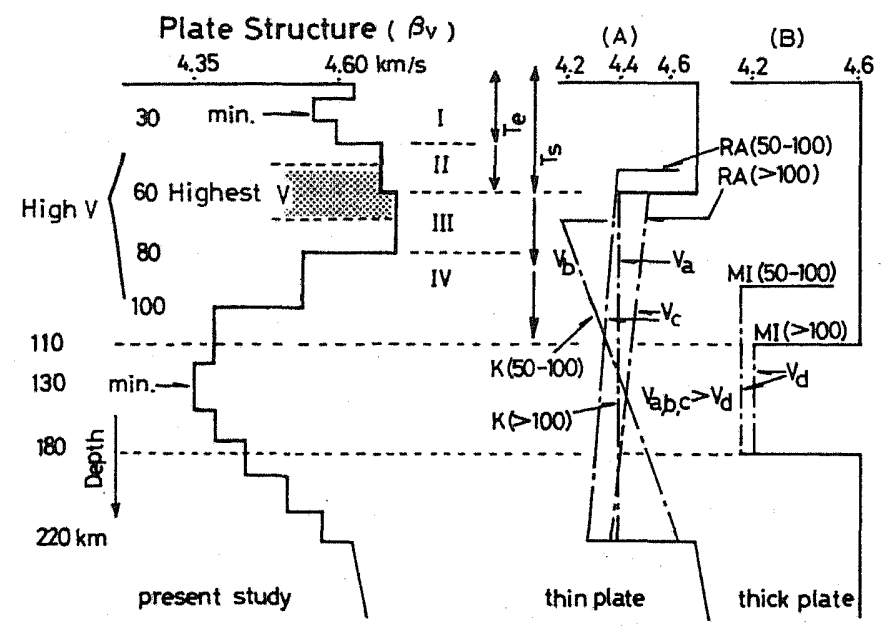

Fig. 8. Schematic representation of the profiles showing the vertically heterogeneous $\beta_{\mathrm{V}}$ structure in the region $>100 \mathrm{~m}$.y. obtained from the present study, together with those of the thin (A) and thick (B) plate models. The vertically heterogeneous $\beta_{\mathrm{V}}$ structure is divided into four layers: I, II, III, and IV. The elastic thickness $\left(T_{\mathrm{e}}\right)$ and the seismic thickness $\left(T_{\mathrm{s}}\right)$ are approximated by solid lines with arrows. The structures for the thin models RA (Regan and Anderson, 1984), K (Kawasaki, 1986), and thick models MI (Mitchell and $\mathrm{Yu}, 1980$ ) are plotted for the regions $>100 \mathrm{~m} . \mathrm{y}$. and 50 to $100 \mathrm{~m} . \mathrm{y}$., with the ages in parentheses after their model abbreviations. The shear wave velocities in the LVZ are denoted by $V_{\mathrm{c}}$ for the RA model, by $V_{\mathrm{a}}$ and $V_{\mathrm{b}}$ for the $\mathrm{K}$ model, and by $V_{\mathrm{d}}$ for the MI model near $130 \mathrm{~km}\left(V_{\mathrm{a}, \mathrm{b}, \mathrm{c}}>V_{\mathrm{d}}\right)$.

sharply with depth, especially below depths of $60 \mathrm{~km}$. Hence, the plate thickness inferred from the $\beta_{\mathrm{H}}$ structures is markedly thinner than that from the $\beta_{\mathrm{V}}$ structures. There is a possibility that the $\beta_{\mathrm{H}}$ structure is highly correlated with the elastic thickness inferred from studies of the plate flexure.

\subsection{The $\beta_{\mathrm{v}}$ structures in younger regions}

In order to advance the study of a vertically heterogeneous lithospheric structure, several inversions were carried out for younger regions using the 8099-type starting model, in which only the $\beta_{\mathrm{v}}$ is determined. For this purpose, the data sets of the Rayleigh wave group velocities by Mitchell and Yu (1980) for the regions 50 to $100 \mathrm{~m}$.y. and 20 to $50 \mathrm{~m}$.y. along with data sets by Yoshii (1975) for the regions 50 to $90 \mathrm{~m}$.y. and 20 to 50 m.y. were independently used. The results are shown in Figs. 9 and 10. The e model $(q=5)$ for the region 50 to 90 m.y. in general resembles that for the region 50 to 100 m.y. (Fig. 9(a)), except for layer I near a depth of $30 \mathrm{~km}$. In this isotropic inversion, layer I for the region 50 to $100 \mathrm{~m}$.y., being slightly different from that obtained for the anisotropic inversion (Fig. 7(a)), is not likely to be well determined. It is notable that the $\mathrm{c}(q=3)$ and $\mathrm{d}(q=4)$ models for the region 50 to $100 \mathrm{~m}$.y. (Fig. 9(a)) resemble 

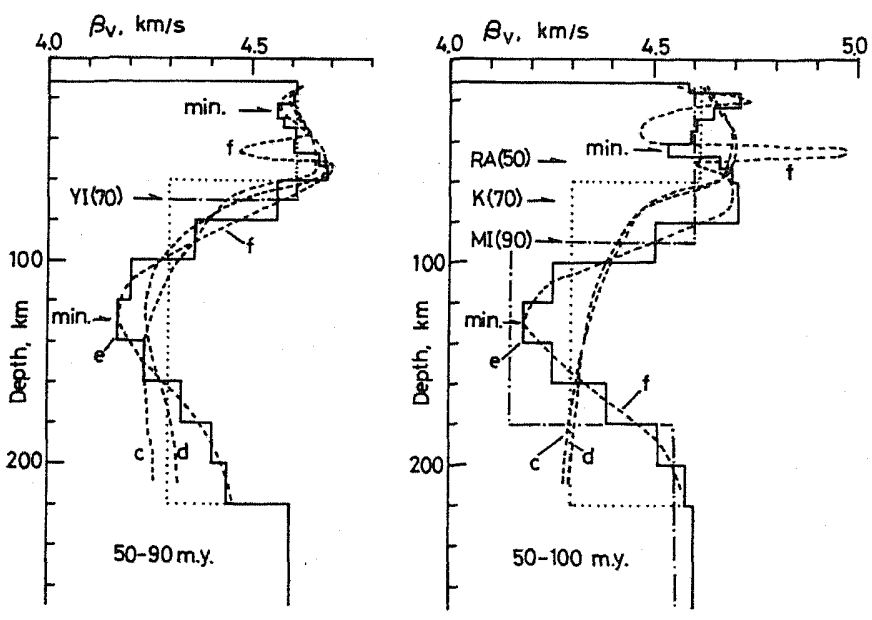

(a)

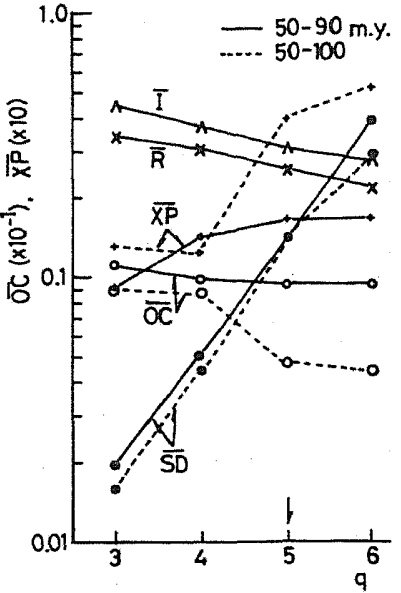

(b)

Fig. 9. (a) Shear wave velocity structures $c, d$, e, and $f$ in the regions 50 to 90 m.y. and 50 to 100 m.y. obtained from Rayleigh wave group velocities, using the 8099-type starting model (dotted lines). (b) Trade-off curves. For the arrows, see the caption in Fig. 2.

the RA model for the same region given in Fig. 8. The trade-off curves (Fig. 9(b)) show that the values of $\overline{O C}$ and $\overline{X P}$ drastically change at $q=5$, and the resultant e model may be an unstable solution for this isotropic inversion.

The $\beta_{\mathrm{v}}$ structures for the region 20 to 50 m.y. are shown in Fig. 10(a), in which the structures for Cases 1 and 2 are obtained using the data sets by Mitchell and $\mathrm{Yu}$ (1980) and Yoshii (1975), respectively. The A and C models denote the simplified lithospheric structure while the B and D models represent the detailed structures. The four structures clearly show that the Characteristic 1 for the region $>100 \mathrm{~m}$.y. (the small-scale LVZ at a depth of $30 \mathrm{~km}$ ) cannot be determined. If the plate bottom is defined as the mid-point between the depth indicating the maximum velocity in the lithosphere and the minimum velocity in the large-scale LVZ, the plate thickness can be estimated as $45 \mathrm{~km}$. The values of $\overline{S D}$ and $\overline{O C}$ taken from the trade-off curves (Fig. 10(b)) for Case 2 are smaller than those for Case 1, due to the difference in the properties of the two data sets.

\section{Discussion}

The structure of the oceanic lithosphere in the Northwestern Pacific was investigated by Asada et al. (1983), by long-range explosion seismological experiments using ocean-bottom seismometers (OBS). The compressional wave velocity structure shows that the bottom of the oceanic lithosphere with a velocity of $8.60 \mathrm{~km} / \mathrm{s}$ is located at a depth of $80 \mathrm{~km}$, and a thin LVZ with a velocity of $8.40 \mathrm{~km} / \mathrm{s}$ at depths between $80.10-120.00 \mathrm{~km}$. The velocity increases from 8.60 to $8.80 \mathrm{~km} / \mathrm{s}$ with depth between 
M. Yoshida
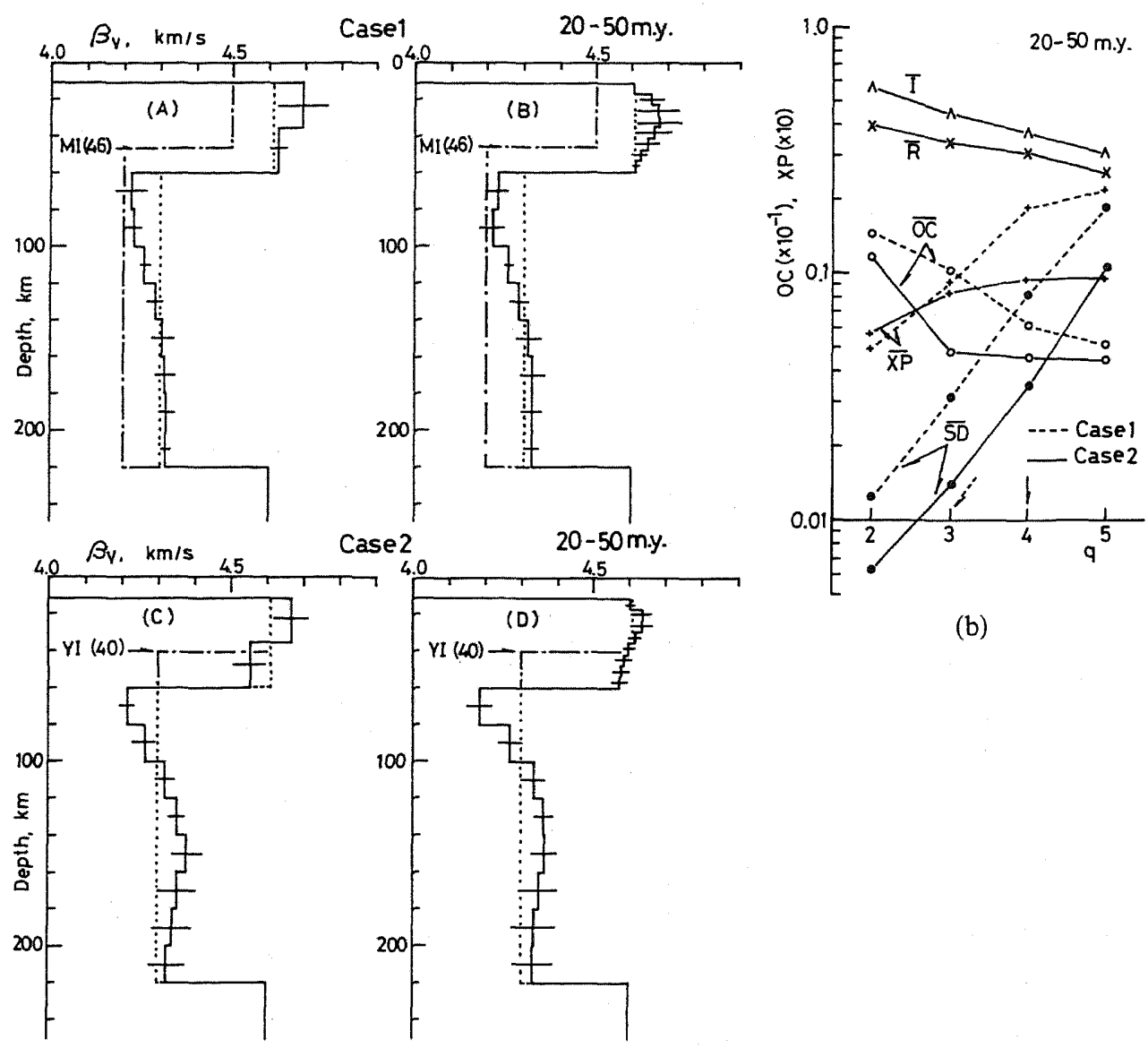

(b)

(a)

Fig. 10. (a) Shear wave velocity structures in the region 20 to $50 \mathrm{~m}$.y. using the 8099-type starting model (dotted lines). The models (A) and (B) are obtained from Case 1 (Rayleigh wave group velocities of Mitchell and Yu, 1980), and the models (C) and (D) from Case 2 (those of Yoshii, 1975). The starting model used by Mitchell and Yu (1980) and the final model obtained by Yoshii (1975) are denoted by chain lines. (b) Trade-off curves for the structures obtained from Case 1 and Case 2. The two arrows on the abscissa indicate that the model (B) for Case 1 is obtained at $q=3$, and the model (D) for Case 2 at $q=4$.

$120.10-300.00 \mathrm{~km}$. This velocity profile is in harmony with the $\beta_{\mathrm{V}}$ structure (Fig. 7(a)) in the region $>100 \mathrm{~m}$.y., inferred from the group velocity. Hence, if the bottom of the vertically heterogeneous lithosphere is defined by Characteristic 2 , the plate thickness of $80 \mathrm{~km}$ inferred from the $\beta_{\mathrm{V}}$ structure agrees with that inferred from the OBS experiments. From OBS long-range explosion experiments, Nagumo et al. (1981) obtained a sub-Moho seismic profile composed of alternating high- and low-velocity 


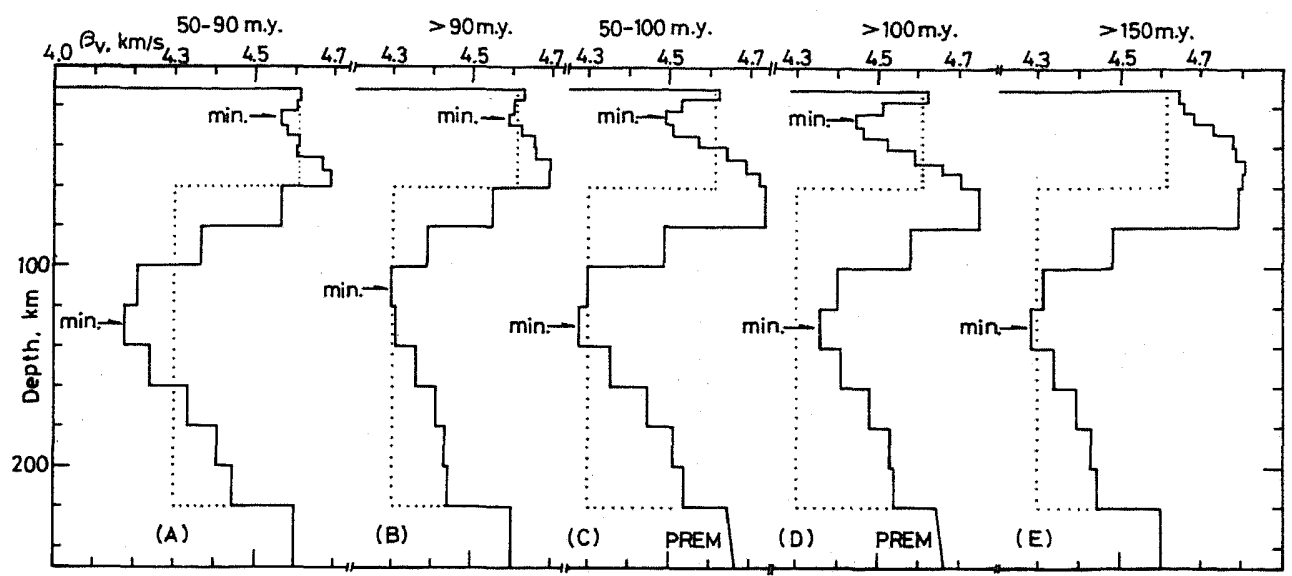

Fig. 11. Shear wave velocity structures in the regions 50 to $170 \mathrm{~m}$.y. The structures (C) and (D) are obtained by anisotropic inversions using the PREM-type starting model and the others by isotropic inversions using the 8099-type starting model.

layers in the Mariana basin, with an age of $>130$ m.y. But the profile is different from the $\beta_{\mathrm{V}}$ structure mentioned above and may suggest a locality in the velocity distribution.

The $\beta_{\mathrm{v}}$ structures, previously obtained for the regions 50 to $170 \mathrm{~m} . \mathrm{y}$. , are shown in Fig. 11. It is clear from this figure that the three characteristics for the region $>100$ m.y. almost always appear. If the heterogeneous plate (D) in the figure, corresponding to the Pacific plate having an age between 110-130 m.y. found near the Japan trench (Sclater et al., 1981), is descending beneath the Japan arcs, Characteristic 1 would produce a difference in rigidity between layers I and II (see also Fig. 8). The solid parts in layer II are likely to form a fracture zone when the exerted tensile stress exceeds the strength of the layer. It seems that the fracture zone corresponds geometrically to the lower seismic plane of the double-planed seismic zone, observed in the regions near the Japan trench (e.g., Umino et al., 1984; Suzuki et al., 1983; Mizoue et al., 1983). However, further study is needed to discuss it more precisely. It will be developed in a separate work.

The $\beta_{\mathrm{V}}$ structure in the region $>100 \mathrm{~m}$.y. obtained in the present work is generally similar to that in the region $>110$ m.y. obtained by Nishimura and Forsyth (1989), who used a different modelling philosophy and data, along with a robust inversion technique (Tarantola and Valette, 1982).

\section{Conclusions}

The structure and thickness of the Pacific plate for the region where the age is greater than $100 \mathrm{~m} . y$. have been investigated by performing several anisotropic and isotropic inversions, making use of the trade-off curves among five quantities calculated for the choice of the optimal solution. The principal results obtained are summarized 
as follows:

(1) The $\beta_{\mathrm{V}}$ structure of the lithosphere consists of four vertically heterogeneous layers and has three characteristics.

(2) Characteristic 1 is that the structure has a small-scale LVZ at a depth of $30 \mathrm{~km}$ in layer I.

(3) Characteristic 2 is that the structure reaches a maximum value at a depth of $70 \mathrm{~km}$ in layer III. Layer II shows a sharp increase of the velocity with depth between $35-60 \mathrm{~km}$. Layer IV between $80-110 \mathrm{~km}$ is a transitional layer with the velocity decreasing downward.

(4) Characteristic 3 is that the structure has a minimum value at a depth of $130 \mathrm{~km}$ in the large-scale LVZ. The velocity increases with depth between 130-220 km.

(5) The vertically heterogeneous structure obtained, being very different from the simple thin and thick plate models, suggests that the bottom of the oceanic lithosphere has to be properly defined in order to precisely define the plate thickness.

(6) The $\beta_{\mathrm{H}}$ is greatest in the uppermost portion of the lithosphere and decreases downward to $150 \mathrm{~km}$. Hence the $\beta_{\mathrm{H}}$ structure does not possess characteristics similar to Characteristics 1 and 2 , and seems to be related to the very thin elastic thickness.

(7) There is a possibility that Characteristic 1 is a factor that may generate the double-planed seismic zone.

The author thanks anonymous reviewers for their comments.

\section{REFERENCES}

Anderson, D. I. and J. Regan, Upper mantle anisotropy and the oceanic lithosphere, Geophys. Res. Lett., 10, 841-844, 1983.

Asada, T., H. Shimamura, S. Asano, K. Kobayasi, and Y. Tomoda, Explosion seismological experiments on long-range profiles in the Northwestern Pacific and the Mariana Sea, Geodynamic Series, Vol. 11, Geodynamics of the Western Pacific Indonesian region, AGU, 105-120, 1983.

Cara, M., Lateral variations of S velocity in the upper mantle from higher mode Rayleigh waves, Geophys. J. R. Astron. Soc., 57, 649-670, 1979.

Dorman; J., M. Ewing, and J. Oliver, Study of shear-velocity distribution in the upper mantle by mantle Rayleigh waves, Bull. Seismol. Soc. Am., 50, 87-115, 1960.

Dziewonski, A. M. and D. L. Anderson, Preliminary reference Earth model, Phys. Earth Planet. Inter., 25, 297-356, 1981.

Ida, Y., Stress and relaxation time in the viscoelastic lithosphere inferred from the outer topographic rise, J. Geophys. Res., 89, 3211-3221, 1984.

Jackson, D. D., Interpretation of inaccurate, insufficient and inconsistent data, Geophys. J. $R$. Astron. Soc., 28, 97-109, 1972.

Kanamori, H. and D. L. Anderson, Importance of physical dispersion in surface wave and free oscillation problems: Review, Rev. Geophys. Space Phys., 15, 105-112, 1977.

Kawasaki, I., Azimuthal anisotropic model of the oceanic upper mantle, Phys. Earth Planet. Inter., 43, 1-21, 1986.

Leeds, A. R., L. Knopoff, and E. G. Kausel, Variations of upper mantle structure under the 
Pacific Ocean, Science, 186, 141-143, 1974.

Liu, H. P., D. L. Anderson, and H. Kanamori, Velocity dispersion due to anelasticity: implications for seismology and mantle composition, Geophys. J. R. Astron. Soc., 47, 41-58, 1976.

Mitchell, B. J., Anelasticity of the crust and upper mantle beneath the pacific Ocean from the inversion of observed surface wave attenuation, Geophys. J. R. Astron. Soc., 46, 521-533, 1976.

Mitchell, B. J. and G. K. Yu, Surface wave dispersion, regionalized velocity models, and anisotropy of the Pacific crust and upper mantle, Geophys. J. R. Astron. Soc., 63, 497-514, 1980.

Mizoue, M., I. Nakamura, H. Chiba, M. Yoshida, H. Hagiwara, and T. Yokota, Westward movement of the seismic activity associated with the earthquake of July 23, 1982 off Ibaraki prefecture, north eastern Honshu, Japan, Bull. Earthq. Res. Inst., Univ. Tokyo, 58, 25-63, 1983.

Nagumo, S., T. Ouchi, J. Kasahara, Y. Tomoda, K. Kobayashi, A. F. Furumoto, M. E. Odegard, and G. H. Sutton, Sub-Moho seismic profile in the Mariana Basin-ocean bottom seismograph explosion experiment, Earth Planet. Sci. Lett., 53, 93-102, 1981.

Nishimura, C. E. and D. W. Forsyth, The anisotropic structure of the upper mantle in the Pacific, Geophys. J., 96, 203-229, 1989.

Regan, J. and D. L. Anderson, Anisotropic models of the upper mantle, Phys. Earth Planet. Inter., 35, 227-263, 1984.

Rodi, W. L., P. Glover, T. M. C. Li, and S. S. Alexander, A fast accurate method for computing group-velocity partial derivatives for Rayleigh and Love waves, Bull. Seismol. Soc. Am., 65, 1105-1114, 1975.

Saito, M., Singular value decomposition and least square method, Geophys. Explor., 36, 36-48, 1983 (in Japanese).

Sclater, J. G., B. Parsons, and C. Jaupart, Oceans and continents: similarities and differences in the mechanisms of heat loss, J. Geophys. Res., 86, 11535-11552, 1981.

Suzuki, S., T. Sasatani, and Y. Motoya, Double seismic zone beneath the middle of Hokkaido, Japan, in the south western side of the Kurile arc, Tectonophysics, 96, 59-76, 1983.

Takeuchi, H. and M. Saito, Seismic surface waves, in Methods in Computational physics, ed. B. A. Bolt, Vol. 11, pp. 217-295, Academic Press, New York, 1972.

Tarantola, A. and B. Valette, Generalized nonlinear inverse problems solved using the least squares criterion, Rev. Geophys. Space Phys., 20, 219-232, 1982.

Umino, N., A. Hasegawa, A. Takagi, S. Suzuki, Y. Motoya, S. Kaneya, K. Tanaka, and Y. Sawada, Focal mechanism of intermediate-depth earthquake beneath Hokkaido and North Honshu, Japan, Zisin, 2, 37, 523-538, 1984 (in Japanese).

Watts, A. B., J. H. Bodine, and M. S. Steckler, Observations of flexure and the state of stress in the oceanic lithosphere, J. Geophys. Res., 85, 6369-6376, 1980.

Wiggins, P. A., The general linear inverse problems: implication of surface and free oscillations for earth structure, Rev. Geophys. Space Phys., 10, 251-285, 1972.

Yoshida, M., Group velocity distributions of Rayleigh waves and two upper mantle models in the Pacific Ocean, Bull. Earthq. Res. Inst., Univ. Tokyo, 53, 319-338, 1978.

Yoshida, M., Group velocity Partial derivatives of Rayleigh and Love waves near the East Pacific Rise: modelling the shear wave velocity structure of the asthenosphere, J. Phys. Earth, 34, $427-440,1986$.

Yoshii, T., Regionality of group velocities of Rayleigh waves in the Pacific and thickening of the plate, Earth Planet. Sci. Lett., 25, 305-312, 1975. 